\title{
Téoros
}

Revue de recherche en tourisme

\section{Penser le tourisme gourmand}

\section{Jean-Pierre Lemasson}

Volume 25, numéro 1, printemps 2006

Entre la culture du goût et le goût de la culture

URI : https://id.erudit.org/iderudit/1071025ar

DOI : https://doi.org/10.7202/1071025ar

Aller au sommaire du numéro

Éditeur(s)

Université du Québec à Montréal

ISSN

0712-8657 (imprimé)

1923-2705 (numérique)

Découvrir la revue

Citer ce document

Lemasson, J.-P. (2006). Penser le tourisme gourmand. Téoros, 25(1), 3-4. https://doi.org/10.7202/1071025ar d'utilisation que vous pouvez consulter en ligne.

https://apropos.erudit.org/fr/usagers/politique-dutilisation/ 


\section{Présentation}

\section{Penser le tourisme gourmand}

\section{Jean-Pierre Lemasson}

Si les liens entre tourisme et gastronomie sont anciens, il existe actuellement une prise de conscience que l'alimentation n'est plus une fonction secondaire de l'activité touristique, mais peut même devenir sa raison centrale. Pour désigner ce changement de perspective, diverses expressions sont utilisées avec plus ou moins de bonheur. Aux États-Unis et au Canada, la notion de tourisme culinaire est largement utilisée (culinary tourism). En Europe, la notion de tourisme gastronomique est parfois utilisée alors que, plus souvent, l'intérêt spécifique porté à un produit, le vin par exemple, fait parler de viti-vini-culture en France ou d'enoturismo en Italie. Dans plusieurs pays, l'agrotourisme est la seule référence mentionnée même si, comme c'est le cas en Angleterre, certains auteurs parlent de tourisme du «bon " goût (tasting tourism). En somme, en rapport à quel référent ce nouveau tourisme se définit-il ? À l'activité culinaire (stricto et largo sensu) ? Aux producteurs ou aux produits? À l'espace rural ou urbain, ou encore au goût, à la sensation du consommateur final? Bref, selon les points de vue, les questions soulevées et les réponses suggérées sont parfois sans point de convergence.

Dans ces conditions, seule la notion de tourisme gourmand nous a paru pertinente pour réconcilier tout à la fois l'intérêt d'une vision holistique et les perspectives qui n'ont finalement de sens que par le plaisir du mangeur. Cette dimension est fondamentale à rappeler, tant les motivations économiques, celles associées à l'offre de produits et services, occultent toutes les autres dimensions possibles d'analyse. Ce numéro de Téoros est donc consacré au tourisme gourmand selon une multiplicité d'angles et de niveaux d'approche. Et ceux présentés

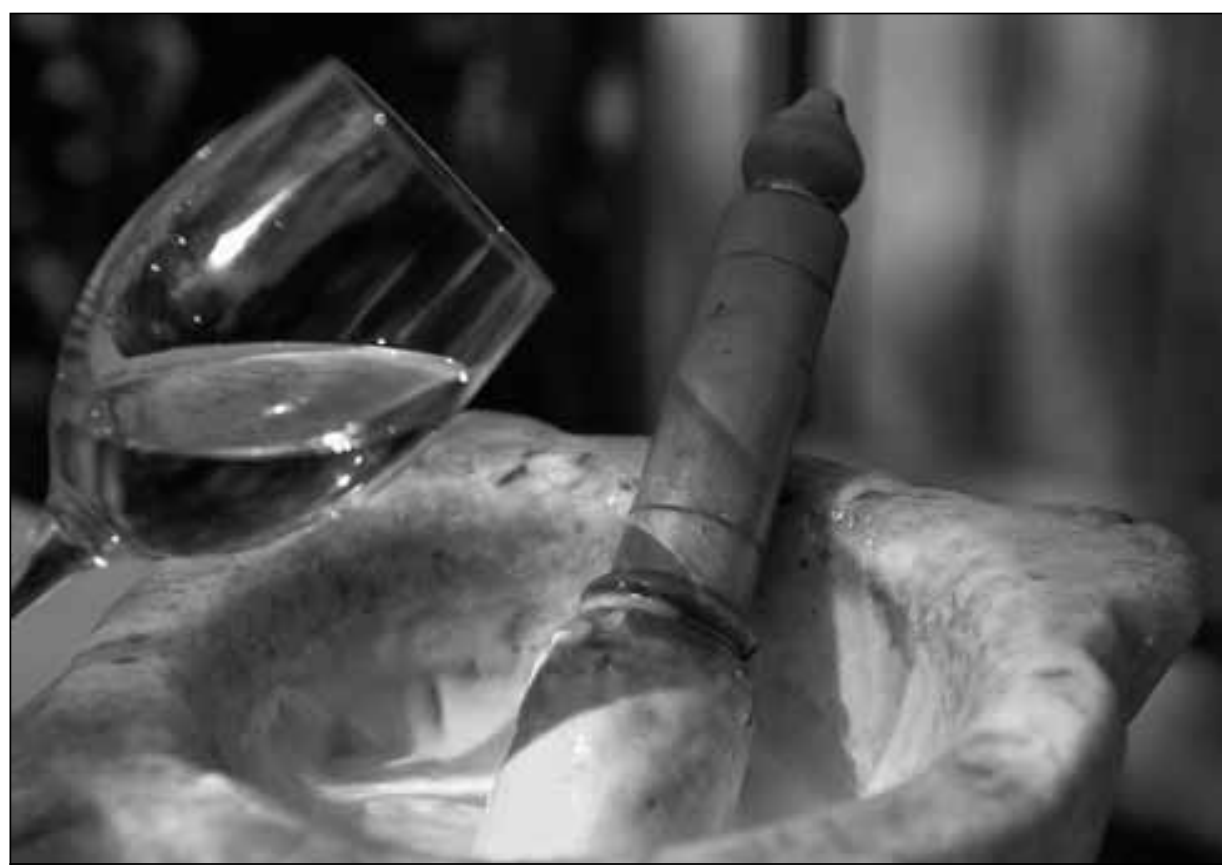

Le mortier, utilisé depuis l'Antiquité, est encore un ustensile de base. II sert entre autres à émulsionner l'aïoli ${ }^{2}$.

Photo : CIVP/F.Millo

n'épuisent pas les perspectives possibles d'analyse. Néanmoins, grâce aux diverses contributions retenues, le lecteur pourra apprécier la richesse d'un champ plus complexe qu'il n'y paraît, puisqu'il ne saurait par ailleurs se dissocier d'enjeux culturels plus vastes. Les dimensions historiques si importantes en Europe comparées à celles d'Amérique du Nord, les niveaux d'analyses macro ou micro sociales, les thèmes privilégiés... démontrent la richesse du sujet qui pourrait sans doute être mieux unifié si davantage de travaux de recherche existaient. En ce sens, nous considérons ce numéro comme une contribution à la mise en place d'un cadre commun de réflexion à partir duquel pourront plus facilement se situer les travaux actuels et futurs dans le domaine.
En premier lieu, Julia Csergo relate les grandes étapes qui, en France, ont conduit tout à la fois à construire la conscience de l'intérêt des produits alimentaires locaux et à les insérer dans un projet politique régionaliste. $\mathrm{Si}$, au XVIIe siècle, le regard des touristes naissant accorde peu d'attention à d'autres nourritures que celle de la cour, peu à peu les voyageurs vont s'intéresser aux particularités alimentaires des pays traversés. Les spécialités gastronomiques sont progressivement reconnues et deviennent des référents identitaires des régions, des symboles forts d'appartenance communautaire, de légitimes marqueurs culturels. La convergence, au début du $X X^{e}$ siècle, des intérêts des professionnels du tourisme et de la restauration avec les aspirations identitaires 
conduira à la création des terroirs et à leur intégration dans une vision patrimoniale. C'est en ce sens qu'il faut comprendre « la monumentalisation » des spécialités alimentaires à laquelle on assiste actuellement.

Gérard Beaudet, quant à lui, pose une question centrale : le tourisme gourmand se définit-il par un espace géographique qui lui est propre? Le terroir se trouve aussi au cœur de son propos comme un carrefour où paysages et produits se rencontrent, un lieu où l'espace et la mémoire se conjuguent et s'ennoblissent mutuellement. Au point où l'on pourrait se demander si le paysage crée du tourisme gourmand ou si c'est le nouveau désir de bien manger et boire qui est source de nouveaux paysages. Cette dernière causalité semble être bien à l'œuvre chez nous, notamment dans le développement récent des vignobles. Dans ces conditions, les nouveaux paysages ne seraient-ils que des espaces de nouvelles formes de marketing alimentaires ou seraient-ils davantage?

Normand Cazelais propose une réflexion sur l'influence de divers guides gastronomiques, tout particulièrement, le guide Michelin, en France. Ceux-ci, qui sont aussi des guides touristiques, ont des effets qui peuvent être profonds tant sur la conception que la qualité de la cuisine des restaurants. Toutefois, aujourd'hui, notamment sous l'influence du mouvement Slow Food, le champ du gastronomique tend à s'élargir à d'autres types de commerce. Serions-nous au début d'une forme de "refondation » des guides gastronomiques?

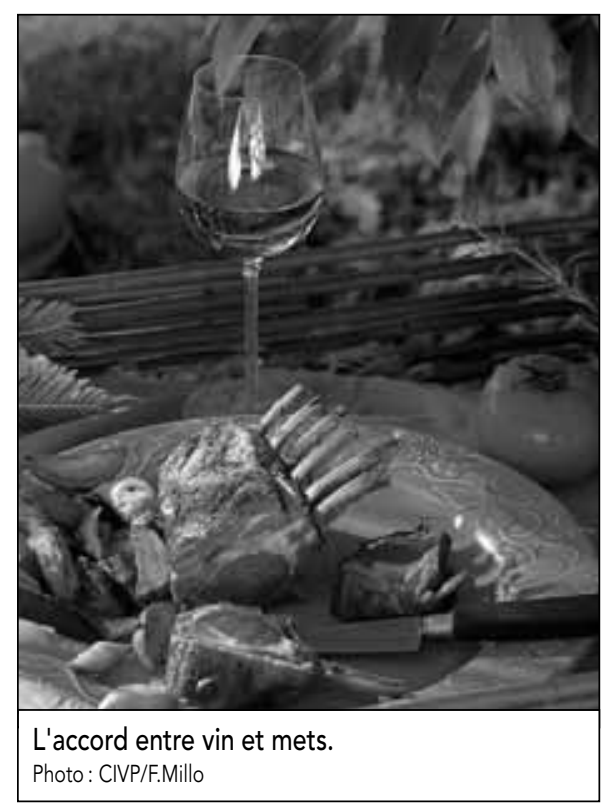

Alberto Capatti, une des figures fondatrices du mouvement Slow Food, nous entraîne, quant à lui, dans l'histoire italienne récente des relations entre tourisme et gastronomie. La fresque qu'il trace, dans un style d'une remarquable légèreté, nous aide à comprendre pourquoi et comment ce pays, qui constitue une véritable référence et un pays pionnier à plusieurs égards, a construit sa réputation actuelle en matière de gastronomie, malgré divers aléas. Son article nous donne la conviction que le tourisme aujourd'hui est un formidable outil pour refonder le patrimoine gastronomique et relancer des productions négligées ou abandonnées.

Les relations entre tourisme et gastronomie ne se jouent pourtant pas que dans l'espace rural. À Montréal, nous assistons à une véritable frénésie culinaire dont l'auteur de ces lignes tentera de saisir la «multidimensionnalité ». Son importance nous conduira à nous demander si aujourd'hui Montréal est de ces villes qui pourraient prétendre à un statut international en matière de bonne chère. À l'heure de la mondialisation du tourisme gourmand, un tel questionnement est incontournable. L'article souligne tout à la fois nos forces considérables, notamment un hédonisme à fleur de langue, pourrait-on dire, mais aussi l'absence d'une volonté politique mobilisatrice. À n'en pas douter nous pourrions mieux faire!

Léonard Dumas, William Menvielle, Jocelyn D. Perreault et Denis Pettigrewdans une perspective plus soucieuse de gestion et de développement, nous invite à constater les difficultés de commercialisation des fromages québécois dont le développement est par ailleurs fulgurant et dont la qualité est désormais attestée par l'obtention de plusieurs prix dans des concours étrangers. Néanmoins, une des difficultés plus particulièrement analysée est l'absence d'une loi définissant clairement les appellations contrôlées, lois dont les effets sur l'agrotourisme ne pourront être que positifs, en aidant les acteurs concernés - producteurs, commerçants et consommateurs - à mieux structurer les options de commercialisation.

À cet égard, l'encadré d'Odette Chaput illustre fort bien les dynamiques de rapprochements entre producteurs et restaurateurs, dynamiques qui s'inscrivent dans une nouvelle conscience régionale, sinon régionaliste, de plus en plus forte chez nous.
Profondément enraciné dans l'espace urbain et abordant des préoccupations souvent formulées par des restaurateurs et des gestionnaires, François Pageau nous ouvre des perspectives nouvelles au regard de l'ambiance comme déterminant de l'expérience gastronomique et facteur d'attractivité. Comme l'a écrit Marc Esquerré du guide GaultMillau, " c'est l'ambiance dorénavant qui crée le lieu. On ne réinvente pas la cuisine, ni les chefs mais la restauration $[\ldots]^{1}$. » On ne saurait mieux dire pour inciter à lire cet article fondé sur une démarche encore exploratoire.

Enfin, le lecteur aura une idée du profil socio-démographique du touriste gourmand canadien dans le résumé du mémoire de maîtrise d'Elena Ignatov de l'université de Waterloo.

Je ne voudrais pas clore cette brève présentation sans faire deux commentaires. En premier lieu, il est bon de rappeler que Téoros avait consacré un numéro en 1984 à la cuisine québécoise, à la restauration et au tourisme. Notre revue a donc de la suite « dans les papilles » et j'ose espérer que les relations entre le tourisme et la gourmandise dans son acception la plus générale soient régulièrement revisitées. En second lieu, ce numéro aura, je l'espère, un effet salutaire sur notre politique du tourisme au Québec, dont la dernière version n'évoque ni la gastronomie ni rien de ce qui l'entoure, comme si les touristes étaient des purs esprits! II y a, me semble-t-il, une grave erreur de perspective considérée soit sous l'angle strictement économique soit, plus largement, sous celui de la qualité de l'expérience de ceux que nous aimerions séduire. Comme si le plaisir n'était pas une affaire sérieuse et le plaisir gourmand une nécessité existentielle!

Jean-Pierre Lemasson est professeur au Département d'études urbaines et touristique à l'Université du Québec à Montréal et rédacteur invité pour ce dossier.

\section{Note}

1 GaultMillau, n 7, avril-mai 2004, p. 32.

2 Pour plus d'information, voir le dictionnaire Larousse Gastronomique, Paris, 1213 p. 